\title{
The Effect of Eight Weeks High Intensity Intermittent Training and Blood Flow Restricted on Angiogenic Markers of Muscle in Male Runners
}

\author{
${ }^{1}$ Seyyed Yaser Alavi *, ${ }^{1}$ Shadmehr Mirdar \\ ${ }^{1}$ Department of Exercise Physiology, Faculty of Sport Sciences, University of Mazandaran, Babolsar, Iran.
}

Submitted 31 December 2019; Accepted in final form 31 March 2020.

\begin{abstract}
Background. The aim of this study was to investigate the effect of eight weeks of HIIT and blood flow restricted (BFR) on protein expressions (VEGF and eNOS) in vastus lateralis of male runners. For this purpose, 15 runners (age: $23 \pm 3$ ) voluntarily participating in this study were divided into three groups: 1) Control 2) HIIT and 3) HIIT+BFR. Methods. The experimental groups were practicing (three sessions a week and six attempts each session for eight weeks). Before and at the end of eight weeks, the biopsy samples were collected from vastus lateralis muscle and the protein expression levels of the VEGF and eNOS were studied by immunohistochemical method. Results. The findings of this study showed that the levels of the VEGF and eNOS were significantly increased in the experimental groups compared to the control group $(\mathrm{p}<0.001)$. There was also a significant difference between experimental groups in the VEGF protein expressions $(\mathrm{p}<0.05)$. Conclusion. In conclusion the HIIT and BFR training can effectively increase the protein expression levels of the VEGF and eNOS in vastus lateralis muscle of runners.
\end{abstract}

KEYWORDS: HIIT, BFR, VEGF, eNOS.

\section{INTRODUCTION}

In recent years, several studies have focused on specific forms of exercise, such as the high intensity intermittent training (HIIT) and blood flow restricted (BFR) that have examined the impact of these exercise models mainly on functional and behavioral Physiological variables (1-3). HIIT are used in the form of vigorous repetition exercises (generally with intensity exceeding $80 \%$ of maximum heart rate), along with alternate recovery periods, as a substitute for traditional endurance training (4). BFR exercises also as a training types via reducing local blood flow, lead to the accumulation of metabolic elements (5). Both the practice models can lead to adaptive responses in long term. Skeletal muscle angiogenesis is one of the adaptation responses that reduce exercise induced stress (6). Angiogenesis is called the formation of new capillaries from existing vessels (7) and is a multifactorial process that can be identified only by understanding the molecular basises (6).

Multiple-fold increase in blood flow during exercise result in increased shear stress and lead to upregulation of eNOSmRNA (nitric oxide synthase mRNA) and protein expression (6). VEGF is also an important angiogenic factor that has been reported following an acute bout of a range of exercise modalities including: submaximal cycling exercise (8), HIIT (9), and sprint interval training (SIT) (10) increased expression at the mRNA levels. The increased expression of VEGF mRNA has been

*. Corresponding Author:

Seyyed Yaser Alavi, $\mathrm{PhD}$

E-mail: yaseralavi@gmail.com 
demonstrated between zero and six hours after the end of exercise, with levels returning to baseline within $20-24$ hours $(11,12)$ and is decreased following a period of training $(12,13)$. In comparison to mRNA expression, the muscle VEGF protein response to an acute bout of exercise and period of training is not well clear. Some reports show following an acute bout of submaximal continuous cycling a decrease (14) or no change in protein levels immediately (15) and up to two hours post exercise (8), whereas, other studies have demonstrated an increase in protein levels 2-4 hours post exercise $(15,16)$. However, in humans no increase in basal VEGF protein content have been reported following 4-8 weeks of either continuous or HIIT $(9,13,17)$, Nevertheless, at one study did identify an increase in VEGF protein following short term (10 days) one legged knee extensor exercise (18). However, there were uncertainties about the impact of a course of HIIT on angiogenic factors yet.

On the other hand, the application of BFR can involve multiple primary contractile stimuli including: shear stress (19), skeletal muscle hypoxia (20), metabolic stress (21) and oxidative stress (22), all of which are important stimuli of angiogenesis. In a recent investigation, the BFR stimulus is applied as an additional stimulus in the recovery intervals during an interval training program (23). In this study 8 trained cyclists undertook one session of SIT either alone or with 2 min of post-exercise BFR during the recovery intervals on ergometer and did not observe any significant differences between two practice methods in VEGF or eNOS mRNA expression post-exercise. In another research, 21 trained male cyclists $\left(\mathrm{V}_{\mathrm{O} 2 \max }=62.8\right.$ ml.min-1.kg-1) undertook 4 weeks of SIT either alone $(n=10)$ or with post-exercise BFR $(n=11)(24)$. The results of this study despite the tendency to increase VEGF levels in the SIT+BFR group, did not reveal any changes in vastus lateralis muscle capillarity. The results of the study by Conceicao et al. (2016) also show that muscle VEGF mRNA expression occurs at a higher level after performing a single session of intense endurance training compared to moderate endurance training with BFR on an ergometer.
Due to the lack of sufficient information about the interactive effects of HIIT and BFR exercises on angiogenic variables of skeletal muscle, it seems that integration these training models can influence skeletal muscle angiogenesis in the long term over the individual application of each of these training methods. Since there is a growing scope for identifying and adopting new compact exercise methods that show greater adaptation responses, therefore the aim of present study was to assess combining HIIT and BFR effect on the immunohistochemical expression of angiogenic markers (VEGF and eNOS) of vastus lateralis in male mid-endurance runners.

\section{MATERIALS AND METHOD}

Study Design. The present applied research, semi-experimental study with inter-group design was conducted on med-endurance runners who were active under the supervision of the athletic board of Amol city-Iran in order to assess the effects of HIIT and HIIT+BFR on angiogenic factors (VEGF and eNOS) in vastus lateralis muscle.

Subjects. All experimental procedures were approved by the Mazandaran University Ethics Approvals (Human Participants) Sub-Committee (IR. UMZ. REC. 1397.048) and conformed in all respects with the Declaration of Helsinki. Participants were fully informed of the risks and discomforts associated with all experimental trials before providing written, informed consent. 15 amateur semi-endurance male runners (age 23 $\pm 3 \mathrm{yr}$ ) that worked in 800 and 1500 materials, volunteered to take part in the study. Inclusion criteria for this study were: having at least two years of training experience, not taking any ergogenic ingredients and supplements for at least six months, be full health and having a BMI below 25. In a pre-training briefing session about the research performing process, advices and recommendations were made and anthropometric measurements of runners including height, weight and limb length were recorded in the Physiology Laboratory of Mazandaran University (Table 1). Then runners were categorized into homogeneous groups through Bruce exhausting test and after recording the records, the runners were divided into three groups $(\mathrm{n}=5)$ of control (Con), HIIT and HIIT + BFR.

Table 1. Anthropometric Characteristics of Runners

\begin{tabular}{ccccccc}
\hline Foot Length & Thigh Environment & Waist Circumference & Subcutaneous Fat Thickness & BMI & Height & Weight \\
\hline $6.5 \pm 102$ & $7.2 \pm 67$ & $4.5 \mathrm{~cm} \pm 92$ & $3.1 \pm 11.2$ & $1.7 \pm 23$ & $5 \mathrm{~cm} \pm 172$ & $4 \mathrm{~kg} \pm 73$ \\
\hline
\end{tabular}


Exercise Training Program. Unlike the control group did not participate in any training programs, experimental groups (HIIT and HIIT + BFR) had to practice the 10-20-30 exercise pattern for eight weeks and three sessions per week. The 10-20-30 training consisted of a 15-min warm-up at a low intensity followed by $6 \times 5$ min running periods interspersed by 2 min of rest. Each 5-min running period consisted of five consecutive 1-min intervals divided into 30,20 , and $10 \mathrm{~s}$ at an intensity corresponding to $\sim 30 \%, \sim 60 \%$, and $\sim 90-100 \%$ of maximal running speed. The difference between the experimental groups was that the HIIT+BFR group had to install $12 \mathrm{~cm}$ wide bands at the upper thighs of both legs when performing the exercise and during the two-minute breaks would put them away.

Experimental Protocol. All of the runners in the experimental group were present in the medical laboratory 72 hours before the start of training and 72 hours after the last exercise session and needle biopsy were taken from them. The control group was sampled only at the first time. At first to perform the needle biopsy, each subject would lie open vault mode. Then the desired position was the external anterior lower third part vastus lateralis muscle, was injected and anesthetized with a syringe containing lidocaine. In the next step, using a biopsy needle, $1.6 \mathrm{~mm}$ slices were removed from the muscle and was rapidly discharged into the formalin-containing falcon tubes. Tissue samples were stored in these tubes for one month and then transferred to histological laboratory for immunohistochemistry studies. After transferring samples to histological laboratory, immunohistochemical expression of VEGF and eNOS proteins by Envision method and using VEGF (code SC-7269 manufactured by
Santa Cruz company of America, the accuracy of measurement $200 \mu \mathrm{g} / \mathrm{ml}$ ) and eNOS (code Ab76198 manufactured by Abcam company of America, the accuracy of measurement 300 $\mu \mathrm{g} / \mathrm{ml})$ specific antibodies were measured.

Statistical Analysis of Data. The Kolmogorov-Smirnov and Levene tests were used to ensure normal distribution of data and equality of error variances, respectively. The covariance analysis test was also used to examine intra-group changes. Statistical analysis was performed using software SPSS. 20 and $\mathrm{p}<0.05$ was considered as the level of significance.

\section{RESULTS}

VGEF. ANCOVA revealed that the effect of groups was significant $\left(\mathrm{f}_{2,11}=70.83, \mathrm{p}<0.001\right.$, $\left.\eta^{2}=0.93\right)$; so that, VEGF increased significantly $(\mathrm{p}<0.001)$ in both HIIT $(47.07 \pm 1.84)$ and HIIT+BFR (53.96 \pm 1.95$)$ groups in comparison with control group (22.92 \pm 1.87$)$. Besides, the difference of HIIT and HIIT+BFR was also significant ( $\mathrm{p}=0.03$ ) (Figure 1).

Immunohistochemical expression of VEGF protein of the vastus lateralis muscle cells have been shown for experimental and control groups (Figure 2).

eNOS. ANCOVA revealed that the effect of groups was significant $\left(f_{2,11}=181.5, p<0.001\right.$, $\left.\Pi^{2}=0.97\right)$; so that, eNOS increased significantly $(\mathrm{p}<0.001)$ in both HIIT $(50.10 \pm 1.11)$ and HIIT+BFR $(51.42 \pm 1.17)$ groups in comparison with control group $(23.34 \pm 1.15)$. But the difference of HIIT and HIIT+BFR was not significant $(\mathrm{p}=0.43)$ (Figure 3$)$.

In Figure 4 has been shown immunohistochemically expression of eNOS protein of the vastus lateralis muscle cells for experimental and control groups.

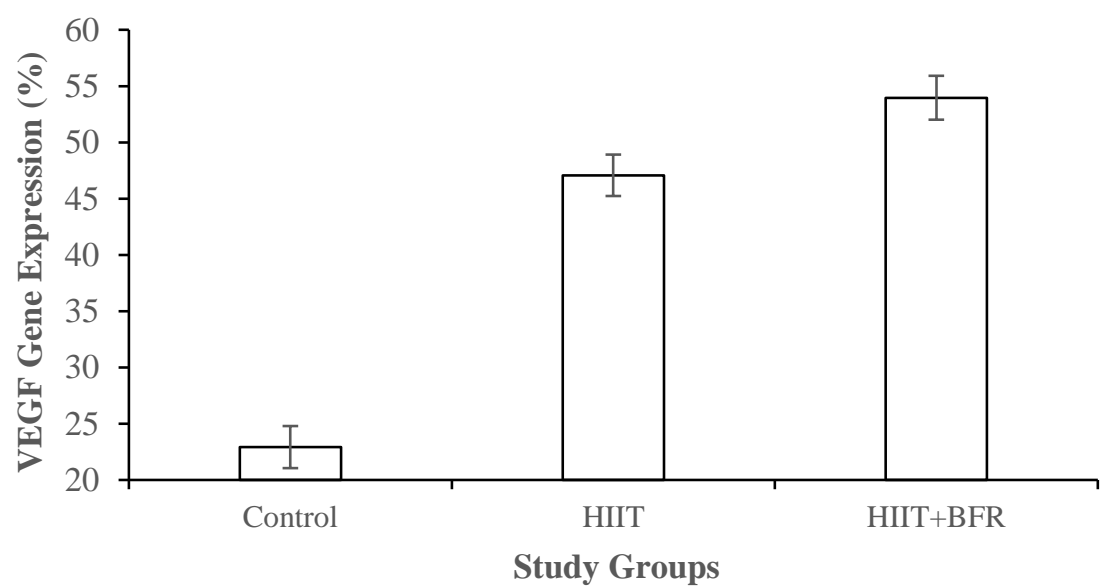

Figure 1. The Effect of 8 Weeks High Intensity IntermittentTraining and Blood Flow Restriction on Muscular VEGF Protein Expression of Male Runners. Covariates Appearing in the Model Are Evaluated at the Following Values: eNOS. preTest=24.8267. HIIT: high intensity intermittent training; BFR: blood flow restriction. "Significant different from Control group at p<0.001. "Significant different from HIIT at p=0.03. 


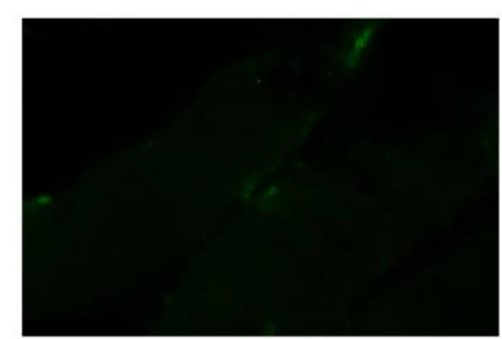

HIIT+BFR

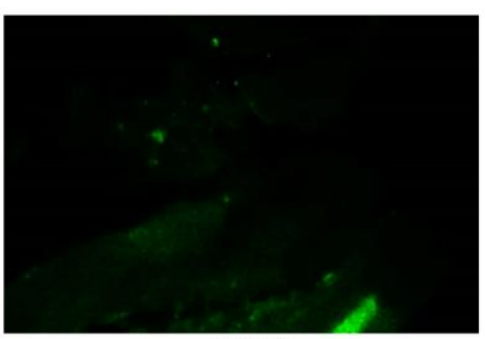

HIIT

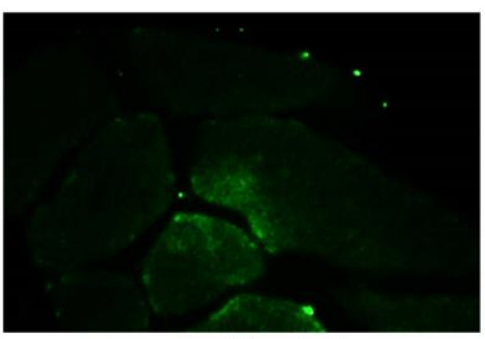

Control

Figure 2. The Effect of 8 Weeks High Intensity Intermittent Training and Blood Flow Restriction on Muscular VEGF Protein Expression of Male Runners. Immunohistochemical Expression of VEGF in Vastus Lateralis Muscle Cells of Experimental and Control Groups. Green Spots Indicate VEGF Protein Expression.

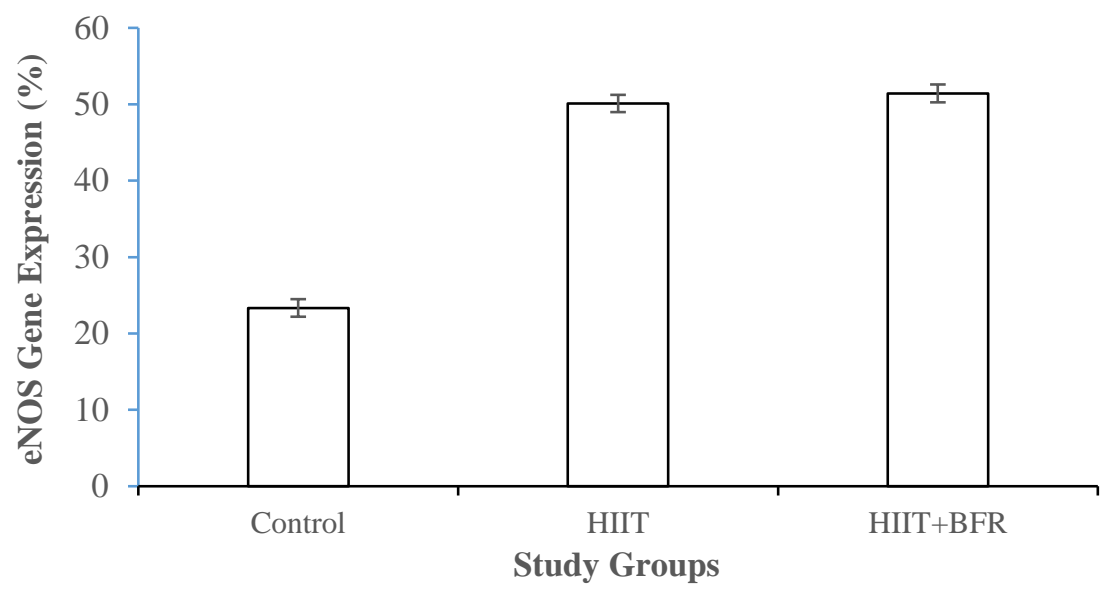

Figure 3. The Effect of 8 Weeks High Intensity Intermittent Training and Blood Flow Restriction on Muscular eNOS Protein Expression of Male Runners. Covariates Appearing in the Model Are Evaluated at the Following Values: eNOS. PreTest=24.8267. HIIT: high intensity intermittent training; BFR: blood flow restriction. "Significant different from Control group at p<0.001.

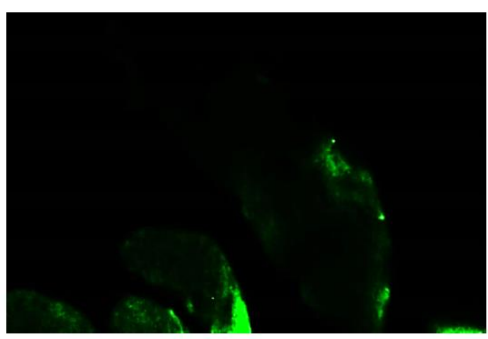

Control

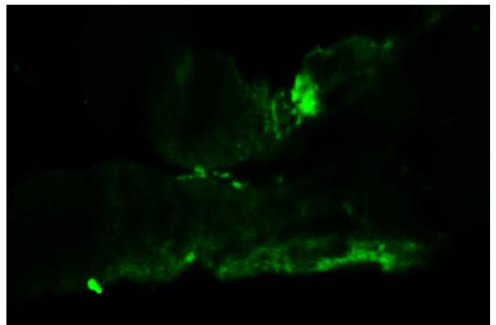

HIIT + BFR

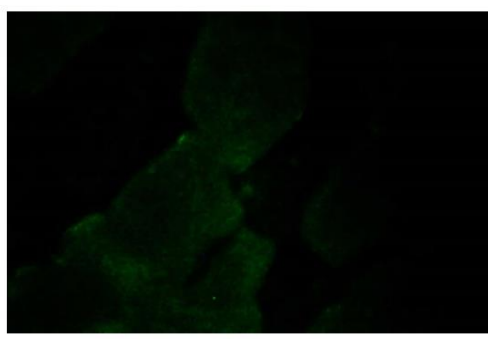

HIIT

Figure 4. The Effect of 8 Weeks High Intensity Intermittent Training and Blood Flow Restriction on Muscular eNOS Protein Expression of Male Runners. Immunohistochemical Expression of eNOS in Vastus Lateralis Muscle Cells of Experimental and Control Groups. Green Spots Indicate VEGF Protein Expression.

\section{DISCUSSION}

The purpose of this study was to investigate the effect of eight weeks of HIIT and BFR training on VEGF and eNOS protein expressions in the vastus lateralis muscle of male medendurance runners. The results of the present study show that the expression of VEGF and eNOS proteins increased significantly after eight weeks of HIIT and HIIT+BFR training compared to the control group. Contrary to the results, Michel et al (2018) did not report any change in the capillarization markers of well-trained cyclist men' vastus lateralis muscles $\left(\mathrm{Vo}_{2 \max }=62.8\right)$ that performed SIT alone or in combination with BFR for four weeks. The inconsistency of the results is probably related to factors such as the level of physical fitness of the subjects, the volume of exercises and the type of exercise protocol. In the study of Michel et al (2018), the training protocol was conducted twice a week for four weeks using cycle ergometer in professional cyclists, whereas in the present study, 10-20-30 training sessions were performed three times a week over eight weeks by amateur med-endurance runners. In humans it is generally considered that 
angiogenesis manifests later than after 4-5 weeks of training (25) and based on the available evidences, exercise training volume or the total amount of time devoted to exercise affects capillary adaptations (6). In the current study, use of BFR exercises was also performed by installing stretch bands during the run training which differs to the mentioned study when applied it between the recoveries of SIT intervals. Taylor et al. (2016b) demonstrated enhanced HIF-1 $\alpha$ mRNA expression following a typical SIT session only when combined with postexercise BFR, however, they did not report any enhancements in either VEGF or eNOS mRNA expression three hours post-exercise with the addition of BFR. The mentioned research suggests that post-exercise BFR is not a sufficient stimulus to enhance transcription of these genes. These findings are in contrast to BFR applied during low load resistance-type or low intensity endurance-type exercise, which has been shown to enhance the acute expression of both PGC- $1 \alpha$ and VEGF mRNA $(2,22)$. The exercise protocols in the aforementioned studies undertaken alone did not elicit increases in VEGF mRNA and only elicited modest increases in PGC- $1 \alpha$ mRNA expression of $\sim 2$-fold, compared to the 5-fold increase following SIT in the study of Taylor et al. (2016b). Therefore, it seems likely that when combined with standard intensity training protocols, which are already potent stimuli of these genes, there is reduced capacity of BFR protocols to further increase the transcriptional response. Exercise induced angiogenesis occurs by a combination of growth factors, hypoxia, shear stress and mechanical stress (6). Studies suggest that angiogenic signals start from within the contracting muscle and PGC- $1 \alpha$ as a master regulator of angiogenesis is involved in this process by interacting to the last nuclear receptor $(E R R \alpha)$. Mechanistically, the functional role of PGC-1 $\alpha$ in VEGF expression in response to exercise training depends on its upstream cascade or P38MAPK (26). Since ROS production is increased during HIIT execution, so it results in more activation of P38MAPK (27) which may eventually lead to more VEGF expression. On the other hand, increased blood flow to the blood vessel bed during exercise increases shear stress, which is a potential stimuli for increased $\mathrm{NO}$ and eNOS expressions $(28,29)$. In consistent to the results of this study, there are studies have assessed the effects of SIT on angiogenic factors
(VEGF and eNOS) (8, 30). Hoier et al. (2013) studied effects of four weeks of SIT on a cycle ergometer in healthy subjects that established to increase VEGFmRNA and eNOS expressions. In the other study, have assessed the structural and endothelial enzymatic changes in skeletal muscle micro-vessels of sixteen young sedentary males in response to ET and SIT for six weeks by Cocks et al. (2013). The expression levels of eNOS increased in both groups, with a significantly greater increase observed following SIT. However, in the present study unlike the VEGF protein values, the difference between the experimental groups (in eNOS protein expression) did not reach a significant level. It seems this discrepancy is related to BFR training which did not induce the adequate amount of shear stress. In other words, the use of flexible bands during HIIT training could not lead to the reactive hyperemia in artery beds when bond pressure was removed during in recoveries. BFR applied during exercise and at rest has been shown to elicit multiple adaptive signals, i.e. increased shear stress, reduced muscle oxygenation and increased markers of oxidative stress, all of which are considered key stimuli of exercise induced angiogenesis and mitochondrial biogenesis $(20,22,31)$. Therefore, although the BFR used in the present study compared with the HIIT alone did not significantly affect the expression of eNOS protein, however, due to the significant increase in VEGF protein expression in the HIIT+ BFR group compared to HIIT, it seems other angiogenic pathways were incrementally affected and evoked.

Studies have demonstrated a greater proliferative effect arising from continuous moderate intensity exercise than intense interval training (9) and a reduction in skeletal muscle VEGF protein content when trained runners increased bouts of intense exercise at the expense of total training volume (17). These investigations suggest that training volume as opposed to training intensity is a greater stimulus for angiogenesis in already trained individuals, at least in the short term. Gliemann (2016) proposed that one reason for a reduction in the angiogenic stimulus with a reduced training volume was the reduction in the shear stress stimulus. Shear stress is a product of both magnitude and duration, but although high levels of shear stress are elicited during intense intervals, it seems likely that this is outweighed by the prolonged stimulus continuous moderate intensity exercise elicits (32). 
In general, angiogenesis is a complex process involving a series of inhibitory-excitatory signaling cascades involved in this process. Therefore, the regulation of angiogenesis is not dependent on one or two factors and there are still need to more exact investigations. In conclusion the results of the present study indicate that an eight-week course of HIIT and HIIT+BFR can increase the expression of the VEGF and eNOS proteins in the vastus lateralis muscle of male med-endurance runners.

\section{APPLICABLE REMARKS}

- The training methods used in this article by increase in muscle protein (VEGF and eNOS) expressions, lead to optimal muscle adaptations that can affect athletes' adaptability to HIIT and HIIT+BFR.

\section{REFERENCES}

1. Garcia-Pinillos F, Soto-Hermoso VM, Latorre-Roman PA. How does high-intensity intermittent training affect recreational endurance runners? Acute and chronic adaptations: A systematic review. J Sport Health Sci. 2017;6(1):54-67. doi: 10.1016/j.jshs.2016.08.010 pmid: 30356547

2. Ferguson RA, Hunt JEA, Lewis MP, Martin NRW, Player DJ, Stangier C, et al. The acute angiogenic signalling response to low-load resistance exercise with blood flow restriction. Eur J Sport Sci. 2018;18(3):397-406. doi: 10.1080/17461391.2017.1422281 pmid: 29343183

3. Mitchell EA, Martin NRW, Turner MC, Taylor CW, Ferguson RA. The combined effect of sprint interval training and postexercise blood flow restriction on critical power, capillary growth, and mitochondrial proteins in trained cyclists. J Appl Physiol (1985). 2019;126(1):51-59. doi: 10.1152/japplphysiol.01082.2017 pmid: 30335575

4. MacInnis MJ, Gibala MJ. Physiological adaptations to interval training and the role of exercise intensity. J Physiol. 2017;595(9):2915-2930. doi: 10.1113/JP273196 pmid: 27748956

5. Spranger MD, Krishnan AC, Levy PD, O'Leary DS, Smith SA. Blood flow restriction training and the exercise pressor reflex: a call for concern. Am J Physiol Heart Circ Physiol. 2015;309(9):H1440-1452. doi: 10.1152/ajpheart.00208.2015 pmid: 26342064

6. Olfert IM, Baum O, Hellsten Y, Egginton S. Advances and challenges in skeletal muscle angiogenesis. Am J Physiol Heart Circ Physiol. 2016;310(3):H326-336. doi: 10.1152/ajpheart.00635.2015 pmid: 26608338

7. Egginton S. Physiological factors influencing capillary growth. Acta Physiol (Oxf). 2011;202(3):225-239. doi: 10.1111/j.1748-1716.2010.02194.x pmid: 20946238

8. Hoier B, Prats C, Qvortrup K, Pilegaard H, Bangsbo J, Hellsten Y. Subcellular localization and mechanism of secretion of vascular endothelial growth factor in human skeletal muscle. FASEB J. 2013;27(9):3496-3504. doi: 10.1096/fj.12-224618 pmid: 23709615

9. Hoier B, Passos M, Bangsbo J, Hellsten Y. Intense intermittent exercise provides weak stimulus for vascular endothelial growth factor secretion and capillary growth in skeletal muscle. Exp Physiol. 2013;98(2):585-597. doi: 10.1113/expphysiol.2012.067967 pmid: 22962287

10. Taylor CW, Ingham SA, Hunt JE, Martin NR, Pringle JS, Ferguson RA. Exercise duration-matched interval and continuous sprint cycling induce similar increases in AMPK phosphorylation, PGC-1alpha and VEGF mRNA expression in trained individuals. Eur J Appl Physiol. 2016;116(8):1445-1454. doi: 10.1007/s00421-016-3402-2 pmid: 27251406

11. Hiscock N, Fischer CP, Pilegaard H, Pedersen BK. Vascular endothelial growth factor mRNA expression and arteriovenous balance in response to prolonged, submaximal exercise in humans. Am J Physiol Heart Circ Physiol. 2003;285(4):H1759-1763. doi: 10.1152/ajpheart.00150.2003 pmid: 12763746

12. Jensen L, Pilegaard H, Neufer PD, Hellsten Y. Effect of acute exercise and exercise training on VEGF splice variants in human skeletal muscle. Am J Physiol Regul Integr Comp Physiol. 2004;287(2):R397-402. doi: 10.1152/ajpregu.00071.2004 pmid: 15117722

13. Hoier B, Nordsborg N, Andersen S, Jensen L, Nybo L, Bangsbo J, et al. Pro- and anti-angiogenic factors in human skeletal muscle in response to acute exercise and training. $J$ Physiol. 2012;590(3):595-606. doi: 10.1113/jphysiol.2011.216135 pmid: 22155930

14. Gavin TP, Robinson CB, Yeager RC, England JA, Nifong LW, Hickner RC. Angiogenic growth factor response to acute systemic exercise in human skeletal muscle. J Appl Physiol (1985). 2004;96(1):19-24. doi: 10.1152/japplphysiol.00748.2003 pmid: 12949011

15. Rullman E, Rundqvist H, Wagsater D, Fischer H, Eriksson P, Sundberg CJ, et al. A single bout of exercise activates matrix metalloproteinase in human skeletal muscle. J Appl Physiol (1985). 2007;102(6):2346-2351. doi: 10.1152/japplphysiol.00822.2006 pmid: 17255365

16. Ryan NA, Zwetsloot KA, Westerkamp LM, Hickner RC, Pofahl WE, Gavin TP. Lower skeletal muscle capillarization and VEGF expression in aged vs. young men. J Appl Physiol (1985). 2006;100(1):178-185. doi: 10.1152/japplphysiol.00827.2005 pmid: 16166239 
17. Gliemann L, Gunnarsson TP, Hellsten Y, Bangsbo J. 10-20-30 training increases performance and lowers blood pressure and VEGF in runners. Scand J Med Sci Sports. 2015;25(5):e479-489. doi: 10.1111/sms.12356 pmid: 25439558

18. Gustafsson T, Knutsson A, Puntschart A, Kaijser L, Nordqvist AC, Sundberg CJ, et al. Increased expression of vascular endothelial growth factor in human skeletal muscle in response to short-term one-legged exercise training. Pflugers Arch. 2002;444(6):752-759. doi: 10.1007/s00424-002-0845-6 pmid: 12355175

19. Paiva FM, Vianna LC, Fernandes IA, Nobrega AC, Lima RM. Effects of disturbed blood flow during exercise on endothelial function: a time course analysis. Brazilian J Med Biol Res. 2016;49:e5100. doi: 10.1590/1414431X20155100

20. Karabulut M, McCarron J, Abe T, Sato Y, Bemben M. The effects of different initial restrictive pressures used to reduce blood flow and thigh composition on tissue oxygenation of the quadriceps. J Sports Sci. 2011;29(9):951958. doi: 10.1080/02640414.2011.572992 pmid: 21547832

21. Takarada Y, Sato Y, Ishii N. Effects of resistance exercise combined with vascular occlusion on muscle function in athletes. Eur J Appl Physiol. 2002;86(4):308-314. doi: 10.1007/s00421-001-0561-5 pmid: 11990743

22. Christiansen D, Murphy RM, Bangsbo J, Stathis CG, Bishop DJ. Increased FXYD1 and PGC-1alpha mRNA after blood flow-restricted running is related to fibre type-specific AMPK signalling and oxidative stress in human muscle. Acta Physiol (Oxf). 2018;223(2):e13045. doi: 10.1111/apha.13045 pmid: 29383885

23. Taylor CW, Ingham SA, Ferguson RA. Acute and chronic effect of sprint interval training combined with postexercise blood-flow restriction in trained individuals. Exp Physiol. 2016;101(1):143-154. doi: 10.1113/EP085293 pmid: 26391312

24. Conceicao MS, Chacon-Mikahil MP, Telles GD, Libardi CA, Junior EM, Vechin FC, et al. Attenuated PGC-1alpha Isoforms following Endurance Exercise with Blood Flow Restriction. Med Sci Sports Exerc. 2016;48(9):16991707. doi: 10.1249/MSS.0000000000000970 pmid: 27128665

25. Jensen L, Bangsbo J, Hellsten Y. Effect of high intensity training on capillarization and presence of angiogenic factors in human skeletal muscle. J Physiol. 2004;557(Pt 2):571-582. doi: 10.1113/jphysiol.2003.057711 pmid: 15020701

26. Chinsomboon J, Ruas J, Gupta RK, Thom R, Shoag J, Rowe GC, et al. The transcriptional coactivator PGC-1alpha mediates exercise-induced angiogenesis in skeletal muscle. Proc Natl Acad Sci U S A. 2009;106(50):21401-21406. doi: 10.1073/pnas.0909131106 pmid: 19966219

27. Hudlicka O, Brown M, Egginton S. Angiogenesis in skeletal and cardiac muscle. Physiol Rev. 1992;72(2):369417. doi: 10.1152/physrev.1992.72.2.369 pmid: 1372998

28. Hudlicka O, Brown MD. Adaptation of skeletal muscle microvasculature to increased or decreased blood flow: role of shear stress, nitric oxide and vascular endothelial growth factor. J Vasc Res. 2009;46(5):504-512. doi: 10.1159/000226127 pmid: 19556804

29. Higashi Y, Yoshizumi M. Exercise and endothelial function: role of endothelium-derived nitric oxide and oxidative stress in healthy subjects and hypertensive patients. Pharmacol Ther. 2004;102(1):87-96. doi: 10.1016/j.pharmthera.2004.02.003 pmid: 15056500

30. Cocks M, Shaw CS, Shepherd SO, Fisher JP, Ranasinghe AM, Barker TA, et al. Sprint interval and endurance training are equally effective in increasing muscle microvascular density and eNOS content in sedentary males. $J$ Physiol. 2013;591(3):641-656. doi: 10.1113/jphysiol.2012.239566 pmid: 22946099

31. Gundermann DM, Fry CS, Dickinson JM, Walker DK, Timmerman KL, Drummond MJ, et al. Reactive hyperemia is not responsible for stimulating muscle protein synthesis following blood flow restriction exercise. J Appl Physiol (1985). 2012;112(9):1520-1528. doi: 10.1152/japplphysiol.01267.2011 pmid: 22362401

32. Gliemann L. Training for skeletal muscle capillarization: a Janus-faced role of exercise intensity? Eur J Appl Physiol. 2016;116(8):1443-1444. doi: 10.1007/s00421-016-3419-6 pmid: 27342704 